\title{
The Effect of Pre-analytical Handling on the Stability of Fractalkine, Monocyte Chemoattractant Protein 1 (MCP-1), Interleukin 6 and Interleukin 8 in Samples of Human Cerebrospinal Fluid: A Prospective Cohort Study.
}

Winnie Charlotte Mortensen ( $\square$ winnie.charlotte.pedersen.mortensen@rsyd.dk )

Odense Universitetshospital https://orcid.org/0000-0002-9659-7125

\section{Laila Bendix}

Odense University Hospital: Odense Universitetshospital

Hanne Irene Jensen

Vejle Hospital: Sygehus Lillebalt Vejle Sygehus

Claus Varnum

Vejle Hospital: Sygehus Lillebalt Vejle Sygehus

Lasse Enkebølle Rasmussen

Vejle Sygehus: Sygehus Lillebalt Vejle Sygehus

Joergen Lauridsen

Syddansk Universitet

Nis Borbye-Lorenzen

SSI: Statens Serum Institut

Kristin Skogstrand

SSI: Statens Serum Institut

Palle Toft

Odense University Hospital: Odense Universitetshospital

Henrik Bjarke Vaegter

Odense University Hospital: Odense Universitetshospital

Morten Rune Blichfeldt-Eckhardt

Odense University Hospital: Odense Universitetshospital

\section{Research}

Keywords: Pre-analytical handling, Time delay, Storage temperature, CX3CL1 (C-X3-C motif chemokine ligand 1), CCL-2 (C-C motif chemokine ligand 2), IL-6, IL-8 
Posted Date: October 27th, 2020

DOl: https://doi.org/10.21203/rs.3.rs-95406/v1

License: (c) (i) This work is licensed under a Creative Commons Attribution 4.0 International License. Read Full License 


\section{Abstract}

\section{Background}

Cytokine networks in cerebrospinal fluid (CSF) are important to our understanding of several neuroinflammatory diseases. Knowledge about optimal handling of samples is limited but important to minimize bias and reduce costs in CSF biomarker studies. The aim of this study was to examine the effect of storage temperature and time delay from CSF sample collection until freezing on the concentration levels of 11 different cytokines thought to be associated with chronic pain.

\section{Methods}

CSF samples from 21 individuals undergoing hip or knee arthroplasty under spinal anesthesia were divided between two tubes. One tube was stored and centrifuged (within 30 minutes) at room temperature, and one tube was stored in ice water and centrifuged (within 30 minutes) at $4^{\circ} \mathrm{C}$. Each tube was split into six vials that were frozen at $-80^{\circ} \mathrm{C}, 0.5,1,2,3,4$, or 5 hours after collection. Cytokines were analyzed using a multiplex panel. A random effect panel data regression was conducted for each biomarker including the variables of storage temperature until freezing and time delay.

Results

Four cytokines had detectable levels: Fractalkine, monocyte chemoattractant protein 1(MCP-1), interleukine 6 (IL-6), and interleukine 8 (IL-8). There was no significant effect of storage temperature and time delay on MCP-1, IL-6, or IL-8 levels. Fractalkine levels showed no clear trend. No concentration differences were observed between samples kept in ice water and those at room temperature except at the 3-hour time point, and there was no overall significant effect of time delay on fractalkine concentration.

\section{Conclusions}

We found no clear effect of storage temperature and time delay up to five hours from sample collection until freezing on the CSF concentrations of fractalkine, MCP-1, IL-6, or IL-8.

Trial registration

Not applicable as the study was not an intervention study.

\section{Background}

Studies of cytokine networks in cerebrospinal fluid (CSF) are important to our understanding of a number of neuroinflammatory diseases such as multiple sclerosis and encephalitides and also possibly of proteopathies and chronic pain conditions where an increasing number of studies indicate neuroimmune pathology (1-5). 
Correct and standardized handling of samples is important to minimize bias and to allow comparison between studies on cytokine biomarkers in the CSF, especially when samples are collected at different centers and settings. While the importance of some confounding factors such as blood contamination, long term storage temperature, repeated freezing, and thawing cycles are well known, we have limited knowledge about other confounders such as time delay and optimal storage temperature from sample collection to spinning and freezing (6-9).

While several studies have demonstrated that both time delay and storage temperature from withdrawal to spinning of blood samples can affect the stability of several cytokines, less is known about the stability of cytokines in CSF samples $(6,10,11)$. Studies conducted at the level of the CSF proteome have shown varying results. Although the human MALDI-TOF proteome and gas chromatography proteome demonstrated stability at room temperature for up to 5 hours (12-15), the human SELDI-TOF proteome was shown to have significant changes in its peptide profile after 4 hours at room temperature and 6-8 hours at $4^{\circ} \mathrm{C}(16)$. In a study of porcine CSF, significant changes in MALDI-FT-ICR proteome and metabolites were reported between 30 and 120 min after collection in samples that had not been centrifuged (17). This has led to the current recommendations for biobanking of CSF to keep the samples at room temperature until spinning and immediate freezing and to minimize the time delay to centrifuging and freezing to 30-60 minutes (maximum 2 hours) after sample collection (7).

The aim of this study was to examine the effect of time delay and storage temperature from sample collection to freezing on the CSF concentration of 11 cytokines that have been associated with chronic pain. We hypothesized that time delay would affect the concentrations of several of the biomarkers and that early freezing would reduce any concentration changes induced by the time delay.

\section{Materials And Methods}

\section{Participants and design}

Participants were included from a larger study, the Odense PAin Research Biobank (OPAR biobank)(18). Eligible participants from the OPAR study were patients with chronic pain due to osteoarthritis who were scheduled for primary total hip arthroplasty or knee arthroplasty under spinal anesthesia at the Department of Orthopedic Surgery and Anesthesia, Lillebaelt Hospital, Denmark. Only patients who were scheduled as the first patient on the day's operation list were included.

The Regional Ethics Committee (S-20180003) and the Danish Data Protection Agency (17/3391) approved this prospective cohort study, and written informed consent was obtained from all participants.

\section{Sample collection and preparation}

Lumbar puncture was performed after departmental standards. CSF was collected during the induction of spinal anesthesia, just before injection of local anesthetic. After collection of CSF for OPAR, additional 5 $\mathrm{ml}$ CSF was collected in a tube and immediately divided into two tubes of $2.5 \mathrm{ml}$ each. One tube was 
placed in ice water and the other tube was kept at room temperature. The samples were immediately transported to the lab where they were centrifuged 30 minutes after withdrawal at $2000 \mathrm{~g}$ for $10 \mathrm{~min}$.

The samples stored in ice water were centrifuged at $4^{\circ} \mathrm{C}$ and divided into six vials of $400 \mu$. The division took place on ice blocks. The vials were stored in ice water for $0.5,1,2,3,4$, or 5 hours after collection, after which they were frozen at $-80^{\circ} \mathrm{C}$ until analysis. The samples stored at room temperature were centrifuged at room temperature and likewise stored for $0.5,1,2,3,4$, or 5 hours after collection before being frozen at $-80^{\circ} \mathrm{C}$ until analysis.

\section{Sample analysis}

The following cytokines were analyzed: interleukin $1 \beta$ (IL-1ß), interleukin 4 (IL-4), interleukin 6 (IL-6), interleukin 8 (IL-8), interleukin 10 (IL-10), interleukin 18 (IL-18), fractalkine (CX3CL1), brain-derived neurotrophic factor (BDNF), high mobility group protein box 1 (HMGB1), tumor necrosis factor (TNF), and monocyte chemoattractant protein 1 (MCP-1).

CSF was analyzed undiluted with in-house developed multiplex immunoassay $(19,20)$ using U-plex plates (Meso-Scale Diagnostics (MSD), Maryland, US) coated with biotinylated antibodies specific for IL$1 \beta$, IL-4, IL-6, IL-8, IL-10, IL-18, TNF, MCP-1, and BDNF on one plate and HMGB1 on another using $0.5 \%$ bovine serum albumin (\#4503, Sigma-Aldrich, Merck Life Science, Søborg, Denmark) in phosphatebuffered saline as assay buffer (see antibody and calibrator details in additional file 1). Biotinylation (using EZ-Link Sulfo-NHS-LC-Biotin \#21327, Thermo Fisher Scientific) was done in-house (if not already biotinylated at purchase) and detection antibodies were SULFO-tagged (R91AO, MSD), both at a challenge ratio of 20:1. The biotinylated antibodies were diluted to $10 \mu \mathrm{g} / \mathrm{mL}$, bound to different linkers 110 (MSD), and added to each plate well according to the manufacturer's instructions. $25 \mu \mathrm{L}$ CSF was added to each well, incubated for 2 hours at room temperature, and then washed. Analyte-specific secondary antibody at $1 \mu \mathrm{g} / \mathrm{mL}$ was added followed by incubation for 2 hours at room temperature. After washing, 2x Read Buffer T (R92TC, MSD) was added, and the plates were immediately read on the QuickPlex SQ 120 (MSD).

Analyses for fractalkine were performed using a commercial kit (K151VCK-1, MSD) following the supplier's instructions. Analyte concentrations were calculated from the calibrator curves on each plate using 4PL logistic regression using the MSD Workbench software.

\section{Statistical analysis}

Because we were unable to find published data on the effect of storage temperature and time delay from sampling to freezing on the individual cytokines examined in this study, a formal study power calculation could not be conducted. We estimated that inclusion of 21 patients would be sufficient. A random effect panel data regression was conducted for each biomarker including the variables storage temperature until freezing, and time delay as an indicator for each time point. Plate number for the multiplex immunoassay was also included in the regression model to control for plate variability. A p-value $<0.05$ 
was considered statistically significant. Biomarker concentrations below detection limit were substituted with a value calculated as lower detection limit/2.

\section{Results}

Twenty-one participants undergoing total hip arthroplasty $(n=11)$ or knee arthroplasty $(n=10)$ under spinal anesthesia between January and April 2018 were included in the study (Table 1).

\section{Table 1. Demographic data}

\begin{tabular}{|ll|}
\hline Sex, female, no (\%) & N=21 \\
\hline Age, years, mean (sd) & $64(10)$ \\
\hline BMl, mean (sd) & $30(5)$ \\
\hline Total hip arthroplasty, no (\%) & $11(52)$ \\
\hline Knee arthroplasty, no (\%) & $10(48)$ \\
\hline
\end{tabular}

Demographic data. BMI=Body mass index.

For four of the biomarkers, the concentrations were within detectable levels (table 2). For the remaining seven biomarkers, there were too few measurements within detectable levels to make any meaningful statistical analyses (table 3). Thus, the biomarkers IL-6, IL-8, fractalkine and MCP-1 were included in the statistical analyses.

\section{Table 2. Detectable cytokines.}

\begin{tabular}{|llll|}
\hline Cytokines & $\begin{array}{l}\text { Level of } \\
\text { detection }\end{array}$ & $\begin{array}{l}\text { Intra-assay coefficient of } \\
\text { variation (CV\%) }\end{array}$ & $\begin{array}{l}\text { Inter-assay coefficient of } \\
\text { variation (CV\%) }\end{array}$ \\
\hline Fractalkine & $100 \%$ & $2.4 \%$ & $12 \%$ \\
\hline MCP-1 & $100 \%$ & $3.3 \%$ & $21 \%$ \\
\hline IL-6 & $87 \%$ & $6.8 \%$ & $20 \%$ \\
\hline IL-8 & $100 \%$ & $3.9 \%$ & $25 \%$ \\
\hline
\end{tabular}

IL-6 = interleukin 6, IL-8 = interleukin 8, MCP-1= Monocyte Chemoattractant Protein 1,

\section{Table 3. Not detectable cytokines.}




\begin{tabular}{|lll|}
\hline Cytokines & Level of detection & Lower detection limit $(\mathrm{pg} / \mathrm{mL})$ \\
\hline BDNF & $1,6 \%$ & 200 \\
\hline TNF & $2.4 \%$ & 8.51 \\
\hline HMGB1 & $4.0 \%$ & 1806 \\
\hline IL-18 & $20.6 \%$ & 1.34 \\
\hline IL-10 & $0.0 \%$ & 4.29 \\
\hline IL-4 & $1.6 \%$ & 514 \\
\hline IL-1 13 & $4.0 \%$ & 1.34 \\
\hline
\end{tabular}

TNF = tumor necrosis factor. BDNF = brain-derived neurotrophic factor. HMGB1 = high mobility group protein box. IL-1 $\beta=$ interleukin $1 \beta$. IL-4 = interleukin 4 . IL-10 = interleukin 10. IL-18 = interleukin 18.

Changes in biomarker concentration are presented in Figure 1. At baseline (defined as the 0.5 time point), there were no significant differences in the concentrations of any of the biomarkers between samples stored in ice water (ice-samples) and samples stored at room temperature (RT-samples).

For fractalkine (Figure 1A), there were no significant differences between ice-samples and RT-samples except at the 3-hour time point ( $\beta$ : 263 $\pm 93 ; p=0.005$; confidence interval $(\mathrm{Cl}): 79,446)$. There was no clear overall picture in regards to time, but the concentrations for ice-samples were significantly higher than baseline at the 3 -hour time point ( $\beta$ : $325 \pm 66 ; \beta<0.001 ; \mathrm{Cl}: 196,455)$ and the 4 -hour time point $(\beta: 255 \pm 66$; $p<0.001 ; \mathrm{Cl}: 125,384)$ and significantly lower than baseline at the 5 -hour time point $(\beta:-151 \pm 67 ; p=0.025$; $\mathrm{Cl}$ :-282,-19). The concentrations of the RT-samples were significantly lower than baseline at the 1-hour time point ( $\beta$ : $-145 \pm 66 ; \mathrm{p}: 0.03 ; \mathrm{Cl}:-275,-15)$ and significantly higher than baseline at the 4-hour time point ( $\beta: 388 \pm 66 ; p<0.001 ; \mathrm{Cl}: 259,518)$. There were no significant differences between baseline and the 5 -hour time point.

For IL-6, IL-8 and MCP-1 (Figure 1B-1D), no significant differences were observed for either storage temperature or time delay.

\section{Discussion}

In this study, we found no clear effect of time delay and storage temperature from CSF sample collection to sample freezing on the concentration of the biomarkers. We found no significant differences for three of the four detectable biomarkers-neither between ice-samples and RT-samples nor at any of the time points compared to baseline. The results for fractalkine were variable with no clear overall trend, which makes it more plausible that the small differences observed at single time points were due to measurement uncertainty rather than real effects of time delay and storage temperature. 
We have not been able to identify previous studies on human CSF that have investigated the effect of time delay and storage temperature until freezing on individual cytokines. Investigations of the CSF proteome using different methods of mass spectrometry (MS) have mostly found the CSF proteome to be relatively stable with respect to both time delay during the first five hours after collection (which was the time period of our study) and storage temperature until freezing (12-15). However, two studies found significant changes in the CSF proteome during the first 5 hours after collection $(16,17)$, and these changes were slowed down by low storage temperature in the study by Rosenling et al. (16), so the evidence is not unequivocal. Furthermore, it is problematic to draw conclusions about the stability of individual cytokines from MS investigations of the proteome for several reasons. First, cytokines can be difficult to detect by MS because of the small molecular weight and low concentrations of most cytokines (21). Second, cytokines are a diverse group of proteins that differ in their stability under various exposures, including long-term storage temperature and repeated freeze-thawing cycles $(9,10,22)$. Therefore, investigation into the effect of sample handling on the concentration of individual cytokines is important to better understand possible sources of bias in biomarker studies of neuroimmune diseases.

There are some limitations to our study. Seven out of 11 biomarkers were below detection limit and this is a well-described problem in human cytokine studies due to the low concentrations of many cytokines (2325). In addition, all samples were spun after 30 minutes. Since unseparated samples are more likely to be affected by time delay from collection to freezing than separated samples $(10,12)$, studies where samples are left unseparated at different time points until freezing are needed to define the optimal preanalytical conditions for cytokine measurements in CSF.

\section{Conclusion}

We found no clear effects of storage temperature and time delay up to five hours from sample collection until freezing on the concentration s of IL-6, IL-8, fractalkine and MCP-1 in CSF samples. However, we were unable to detect 7 out of 11 biomarkers. More studies of the effect of sample handling on the stability of these and other individual cytokines in CSF are warranted.

\section{Abbreviations}

BDNF: Brain-derived neurotrophic factor

CSF: Cerebrospinal fluid

GDPR: General Data Protection Regulation

HMGB1: High mobility group protein box 1

IL-1 $\beta$ : Interleukin-1 $\beta$

IL-4: Interleukin-4 
IL-6: Interleukin-6

IL-8: Interleukin-8

IL-10: Interleukin-10

IL-18: Interleukin-18

MCP-1: Monocyte chemoattractant protein

MS: Mass spectrometry

RT: Room temperature

TNF: Tumor necrosis factor

\section{Declarations}

\section{Ethics approval and consent to participate}

The Regional Ethics Committee (S-20180003) and the Danish Data Protection Agency (17/3391) approved this study, and written informed consent was obtained from all participants.

\section{Consent for publication}

Not applicable

\section{Availability of data and materials}

The datasets generated and/or analyzed during the current study are not publicly available because it is not possible to publish the research dataset in accordance with applicable legislation in EU and Denmark (GDPR and the Danish Act on Data Protection). To be made public, data must be fully anonymized, which means that it must not be pseudo-anonymized or possible to recognize or trace back the data to individuals, not even by the individuals themselves. As the dataset comes from the Odense PAin Research biobank, full anonymization is not possible, but the data are available from the corresponding author on reasonable request and in accordance with applicable legislation.

\section{Competing interests}

The authors declare that they have no competing interests.

\section{Funding}

The Danish Rheumatism Association (A6088), Aase og Ejnar Danielsens Foundation, and the Development Foundation, Lillebaelt Hospital. 
None of the funders participated in any aspect of design, data collection, analyses, interpretation of data, or writing of the manuscript.

\section{Authors' contributions}

WCPM: Design of the study, data collection and interpretation, drafting the article.

$L B$ : Conception and design of the study, interpretation of data, and revising the article critically for important intellectual content.

HIJ: Design of the study, data collection and interpretation, and revising the article critically for important intellectual content.

$C V$ : Design of the study, data interpretation, and revising the article critically for important intellectual content.

LER: Design of the study, data interpretation, and revising the article critically for important intellectual content.

JTL: Design of the study, data analysis and interpretation, and revising the article critically for important intellectual content.

NBL: Biochemical analysis, interpretation of data, revising the article critically for important intellectual content.

$K S$ : Biochemical analysis, interpretation of data, revising the article critically for important intellectual content.

$P T$ : Design of the study, data interpretation, and revising the article critically for important intellectual content.

$H B V$ : Design of the study, data collection and interpretation, and revising the article critically for important intellectual content.

MRBE: Conception and design of the study, data collection, analysis and interpretation, drafting of article.

All authors approved the final version of the article.

\section{Acknowledgements}

Some of the study data were collected and managed using REDCap electronic data capture tools hosted at OPEN, Open Patient data Explorative Network, Odense University Hospital, Region of Southern Denmark.

Claire Gudex contributed with proofreading. 


\section{References}

1. Becher B, Spath S, Goverman J. Cytokine networks in neuroinflammation. Nat Rev Immunol. 2017;17(1):49-59.

2. Ji RR, Nackley A, Huh Y, Terrando N, Maixner W. Neuroinflammation and Central Sensitization in Chronic and Widespread Pain. Anesthesiology. 2018;129(2):343-66.

3. Ji RR, Berta T, Nedergaard M. Glia and pain: is chronic pain a gliopathy? Pain. 2013;154 Suppl 1:S1028.

4. Ellis A, Bennett DL. Neuroinflammation and the generation of neuropathic pain. $\mathrm{Br} \mathrm{J}$ Anaesth. 2013;111(1):26-37.

5. Lepennetier G, Hracsko Z, Unger M, Van Griensven M, Grummel V, Krumbholz M, et al. Cytokine and immune cell profiling in the cerebrospinal fluid of patients with neuro-inflammatory diseases. $J$ Neuroinflammation. 2019;16(1):219.

6. Teunissen CE, Petzold A, Bennett JL, Berven FS, Brundin L, Comabella M, et al. A consensus protocol for the standardization of cerebrospinal fluid collection and biobanking. Neurology. 2009;73(22):1914-22.

7. Teunissen $\mathrm{CE}$, Tumani H, Engelborghs S, Mollenhauer B. Biobanking of CSF: international standardization to optimize biomarker development. Clin Biochem. 2014;47(4-5):288-92.

8. Otto M, Bowser R, Turner M, Berry J, Brettschneider J, Connor J, et al. Roadmap and standard operating procedures for biobanking and discovery of neurochemical markers in ALS. Amyotroph Lateral Scler. 2012;13(1):1-10.

9. Lee JE, Kim SY, Shin SY. Effect of Repeated Freezing and Thawing on Biomarker Stability in Plasma and Serum Samples. Osong Public Health Res Perspect. 2015;6(6):357-62.

10. Flower L, Ahuja RH, Humphries SE, Mohamed-Ali V. Effects of sample handling on the stability of interleukin 6, tumour necrosis factor-alpha and leptin. Cytokine. 2000;12(11):1712-6.

11. West-Nielsen M, Hogdall EV, Marchiori E, Hogdall CK, Schou C, Heegaard NH. Sample handling for mass spectrometric proteomic investigations of human sera. Anal Chem. 2005;77(16):5114-23.

12. Berven FS, Kroksveen AC, Berle M, Rajalahti T, Flikka K, Arneberg R, et al. Pre-analytical influence on the low molecular weight cerebrospinal fluid proteome. Proteomics Clin Appl. 2007;1(7):699-711.

13. Jimenez CR, Koel-Simmelink M, Pham TV, van der Voort L, Teunissen CE. Endogeneous peptide profiling of cerebrospinal fluid by MALDI-TOF mass spectrometry: Optimization of magnetic beadbased peptide capture and analysis of preanalytical variables. Proteomics Clin Appl. 2007;1(11):1385-92.

14. Bruegel M, Planert M, Baumann S, Focke A, Bergh FT, Leichtle A, et al. Standardized peptidome profiling of human cerebrospinal fluid by magnetic bead separation and matrix-assisted laser desorption/ionization time-of-flight mass spectrometry. J Proteomics. 2009;72(4):608-15.

15. Wuolikainen A, Hedenstrom M, Moritz T, Marklund SL, Antti H, Andersen PM. Optimization of procedures for collecting and storing of CSF for studying the metabolome in ALS. Amyotroph Lateral 
Scler. 2009;10(4):229-36.

16. Ranganathan S, Polshyna A, Nicholl G, Lyons-Weiler J, Bowser R. Assessment of Protein Stability in Cerebrospinal Fluid Using Surface-Enhanced Laser Desorption/lonization Time-of-Flight Mass Spectrometry Protein Profiling. Clin Proteomics. 2006;2(1-2):91-101.

17. Rosenling T, Slim CL, Christin C, Coulier L, Shi S, Stoop MP, et al. The effect of preanalytical factors on stability of the proteome and selected metabolites in cerebrospinal fluid (CSF). J Proteome Res. 2009;8(12):5511-22.

18. The Odense PAin Research(OPAR)-Biobank https://open.rsyd.dk/OpenProjects/openProject.jsp? openNo=341\&lang=da. Accessed 11. October 2020.

19. Soegaard SH, Rostgaard K, Skogstrand K, Wiemels JL, Schmiegelow K, Hjalgrim H. Neonatal Inflammatory Markers Are Associated with Childhood B-cell Precursor Acute Lymphoblastic Leukemia. Cancer Res. 2018;78(18):5458-63.

20. Starhof C, Winge K, Heegaard NHH, Skogstrand K, Friis S, Hejl A. Cerebrospinal fluid proinflammatory cytokines differentiate parkinsonian syndromes. J Neuroinflammation. 2018;15(1):305.

21. Kupcova Skalnikova H, Cizkova J, Cervenka J, Vodicka P. Advances in Proteomic Techniques for Cytokine Analysis: Focus on Melanoma Research. Int J Mol Sci. 2017;18(12).

22. de Jager W, Bourcier K, Rijkers GT, Prakken BJ, Seyfert-Margolis V. Prerequisites for cytokine measurements in clinical trials with multiplex immunoassays. BMC Immunol. 2009;10:52.

23. Backonja MM, Coe CL, Muller DA, Schell K. Altered cytokine levels in the blood and cerebrospinal fluid of chronic pain patients. J Neuroimmunol. 2008;195(1-2):157-63.

24. Backryd E, Tanum L, Lind AL, Larsson A, Gordh T. Evidence of both systemic inflammation and neuroinflammation in fibromyalgia patients, as assessed by a multiplex protein panel applied to the cerebrospinal fluid and to plasma. J Pain Res. 2017;10:515-25.

25. Soderlund J, Olsson SK, Samuelsson M, Walther-Jallow L, Johansson C, Erhardt S, et al. Elevation of cerebrospinal fluid interleukin-1ss in bipolar disorder. J Psychiatry Neurosci. 2011;36(2):114-8.

\section{Figures}




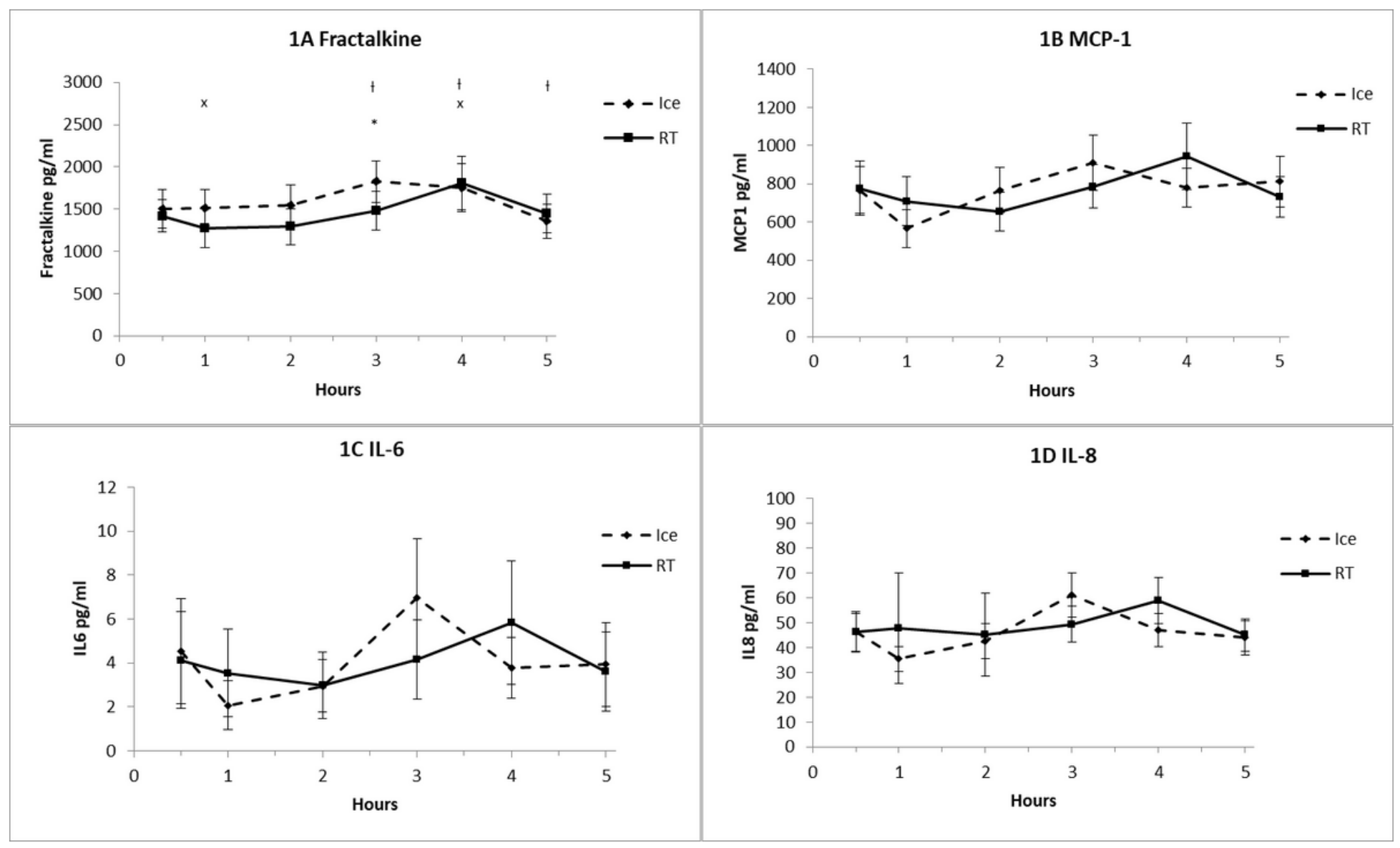

Figure 1

Effects of time delay and storage temperature on cytokine levels Cerebral spinal fluid (CSF) levels of Fractalkine (1A), Monocyte Chemoattractant Protein 1 (MCP-1), Interleukin 8 (IL-8) and Interleukin 6 (IL-6) for samples stored in icewater (Ice) and room at temperature (RT). Data are mean CSF-levels with 95\% Confidence Interval. * Significant difference between levels for Ice-samples and RT-samples; $₫$ significant difference between levels on the Ice-curve and baseline ( 0.5 time point); $x$ significant difference between levels on the RT-curve and baseline ( 0.5 time point). 


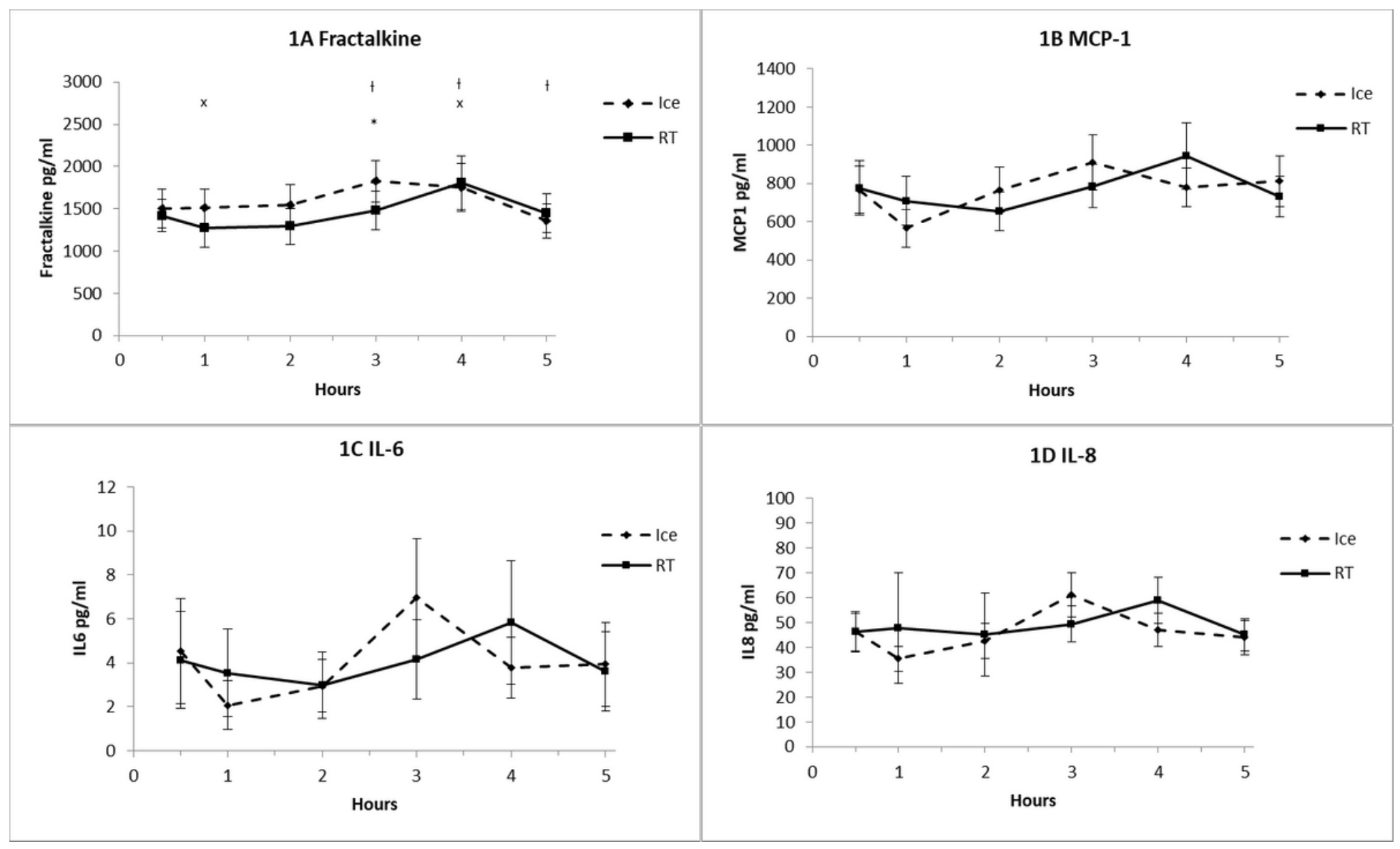

Figure 1

Effects of time delay and storage temperature on cytokine levels Cerebral spinal fluid (CSF) levels of Fractalkine (1A), Monocyte Chemoattractant Protein 1 (MCP-1), Interleukin 8 (IL-8) and Interleukin 6 (IL-6) for samples stored in icewater (Ice) and room at temperature (RT). Data are mean CSF-levels with $95 \%$ Confidence Interval. * Significant difference between levels for Ice-samples and RT-samples; $₫$ significant difference between levels on the Ice-curve and baseline ( 0.5 time point); $x$ significant difference between levels on the RT-curve and baseline ( 0.5 time point).

\section{Supplementary Files}

This is a list of supplementary files associated with this preprint. Click to download.

- Additionalfile11.xlsx

- Additionalfile11.xlsx 